\title{
The NNbar and multihadron production at the threshold at VEPP2000
}

\author{
E. P. Solodov ${ }^{1,2, *}$, R. R. Akhmetshin ${ }^{1,2}, \quad$ A. N. Amirkhanov ${ }^{1,2}, \quad$ A. V. Anisenkov ${ }^{1,2}$, \\ V. M. Aulchenko ${ }^{1,2}$, V. Sh. Banzarov ${ }^{1}$, N. S. Bashtovoy ${ }^{1}$, D. E. Berkaev ${ }^{1,2}$, A. E. Bondar ${ }^{1,2}$,
} A. V. Bragin ${ }^{1}$ ， S. I. Eidelman ${ }^{1,2,5}$ ， D. A. Epifanov ${ }^{1,2}$ ， L. B. Epshteyn ${ }^{1,2,3}$ ， A. L. Erofeev ${ }^{1,2}$, G. V. Fedotovich ${ }^{1,2}, \quad$ S. E. Gayazov ${ }^{1,2}, \quad$ A. A. Grebenuk ${ }^{1,2}, \quad$ S. S. Gribanov ${ }^{1,2}$, D. N. Grigoriev ${ }^{1,2,3}$ ， F. V. Ignatov ${ }^{1,2}$ ， V. L. Ivanov ${ }^{1,2} ， \quad$ S. V. Karpov ${ }^{1} ， \quad$ V. F. Kazanin ${ }^{1,2}$,

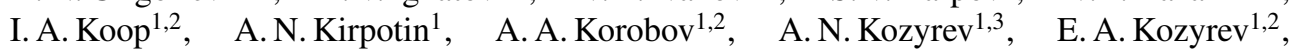
P. P. Krokovny ${ }^{1,2}$, A. E. Kuzmenko ${ }^{1,2}$, A.S. Kuzmin ${ }^{1,2}$, I. B. Logashenko ${ }^{1,2}$, P. A. Lukin ${ }^{1,2}$, K. Yu. Mikhailov ${ }^{1}$, V.S. Okhapkin ${ }^{1}$, Yu. N.Pestov ${ }^{1}$, A.S. Popov ${ }^{1,2}$, G. P. Razuvaev ${ }^{1,2}$, Yu. A. Rogovsky ${ }^{1}$, A. A. Ruban ${ }^{1}$, N. M. Ryskulov ${ }^{1}$, A. E. Ryzhenenkov ${ }^{1,2}$, A. V. Semenov ${ }^{1,2}$, Yu. M. Shatunov ${ }^{1}$, P. Yu. Shatunov ${ }^{1}$, V. E. Shebalin ${ }^{1,2}$, D. N. Shemyakin ${ }^{1,2}$, B. A. Shwartz ${ }^{1,2}$, D. B. Shwartz ${ }^{1,2}$, A. L. Sibidanov ${ }^{1,4}$, V. M. Titov ${ }^{1}, \quad$ A. A. Talyshev ${ }^{1,2}$, A. I. Vorobiov ${ }^{1}$, I. M. Zemlyansky ${ }^{1}$, and Yu. V. Yudin ${ }^{1,2}$

${ }^{1}$ Budker Institute of Nuclear Physics, SB RAS, Novosibirsk, 630090, Russia

${ }^{2}$ Novosibirsk State University, Novosibirsk, 630090, Russia

${ }^{3}$ Novosibirsk State Technical University, Novosibirsk, 630092, Russia

${ }^{4}$ University of Victoria, Victoria, BC, Canada V8W 3P6

${ }^{5}$ Lebedev Physical Institute RAS, Moscow, 119333, Russia

\begin{abstract}
A study of hadron production at the nucleon-antinucleon threshold has been performed with the CMD-3 detector at the VEPP-2000 $e^{+} e^{-}$collider. A very fast rise with an about $1 \mathrm{MeV}$ width has been observed in the $e^{+} e^{-} \rightarrow p \bar{p}$ cross section. A sharp drop in the $e^{+} e^{-} \rightarrow 3\left(\pi^{+} \pi^{-}\right)$cross section has been confirmed and found to have a width of less than $2 \mathrm{MeV}$, in agreement with the observed fast rise of the $e^{+} e^{-} \rightarrow p \bar{p}$ cross section. For the first time a similar sharp drop is demonstrated in the $e^{+} e^{-} \rightarrow K^{+} K^{-} \pi^{+} \pi^{-}$cross section. The behavior of the $e^{+} e^{-} \rightarrow 3\left(\pi^{+} \pi^{-}\right), K^{+} K^{-} \pi^{+} \pi^{-}$cross sections cannot be explained by an interference of any resonance amplitude with continuum, therefore this phenomenon cannot be due to a narrow near-threshold resonance. No such structure has been observed in the $e^{+} e^{-} \rightarrow 2\left(\pi^{+} \pi^{-}\right)$cross section.
\end{abstract}

\section{Introduction}

Production of six pions in $e^{+} e^{-}$annihilation, studied at DM2 [1-3], showed a "dip" in the cross section at about $1.9 \mathrm{GeV}$, confirmed later by the Fermilab E687 experiment in photoproduction $[4,5]$, and with a much larger effective integrated luminosity at BaBar [6] using initial-state radiation (ISR). Even earlier, a narrow structure near the proton-antiproton threshold has been also observed in the total cross section of $e^{+} e^{-}$annihilation into hadrons in the FENICE experiment [7]. A measurement of the CMD-3 Collaboration [8] confirmed these

\footnotetext{
*e-mail: solodov@inp.nsk.su
} 
observations and demonstrated that the drop in the $e^{+} e^{-} \rightarrow 3\left(\pi^{+} \pi^{-}\right)$cross section occurred in the narrow energy range of less than $10 \mathrm{MeV}$ width. The origin of the "dip" remains unclear, but one of the explanations suggests the presence of a below-threshold proton-antiproton $(p \bar{p})$ resonance [9]. Alternatively, in Ref. [10-13] the "dip" is due to the strong interaction in virtual nucleon-antinucleon $(N \bar{N})$ production, and is related to the fast rise of the $e^{+} e^{-} \rightarrow N \bar{N}$ cross section and $N \bar{N}$ annihilation to hadrons. This hypothesis is supported by the fast increase of the $p \bar{p}[14,15]$ and $n \bar{n}$ [16] form factors near threshold, and explains a similar drop in the $\eta^{\prime}(958) \pi^{+} \pi^{-}$spectrum, observed by the BES-III Collaboration in the $J / \psi \rightarrow \eta^{\prime}(958) \pi^{+} \pi^{-} \gamma$ decay [17]. The authors of Ref. [10] consider the two-step process $e^{+} e^{-} \rightarrow N \bar{N} \rightarrow$ multipions and evaluate the total reaction amplitude for various intermediate mechanisms of the $e^{+} e^{-} \rightarrow 5 \pi, 6 \pi$ reactions. In Refs. [11-13] the authors go even further taking into account the proton-neutron mass difference and $\bar{p} p$ Coulomb interaction.

However, the mass-energy resolution of the previous experiments does not allow a study of the fine structure of the "dip" or the rise of the $e^{+} e^{-} \rightarrow N \bar{N}$ cross section. Therefore we decided to repeat a scan of this energy range with a larger data sample and a fine step in an attempt to measure the width of the dip. In this paper we present the analysis of 50 $\mathrm{pb}^{-1}$ of integrated luminosity collected with the CMD-3 detector [18] at 29 center-of-mass (c.m.) energy points at the VEPP-2000 collider with the upgraded injection complex [1922]. While the data have been collected in the $1.5-2.0 \mathrm{GeV}$ center-of-mass energy $\left(\mathrm{E}_{\mathrm{c} . \mathrm{m} .}\right)$ range, the scope of this paper is a detailed study of the $N \bar{N}$ threshold region. The scan of the $N \bar{N}$-threshold energy range was performed with a fine step, corresponding to the c.m. energy spread. The beam energy and energy spread have been monitored by the back-scatteringlaser-light system [23, 24], providing an absolute energy measurement with better than 0.1 $\mathrm{MeV}$ uncertainty in every single measurement. During data taking the $\mathrm{E}_{\mathrm{c} . \mathrm{m}}$. variations around a central value did not exceed $0.1 \mathrm{MeV}$ at each energy point: this value is taken as the systematic uncertainty estimate. The beam energy spread, shown in Fig. 1, is corresponding to the c.m. energy spread $\sigma_{\mathrm{E}_{\mathrm{c} . \mathrm{m} .}}=0.95 \pm 0.10 \mathrm{MeV}$ at the $N \bar{N}$ threshold: the added uncertainty is our estimate of a systematic effect with a negligible contribution of the statistics.
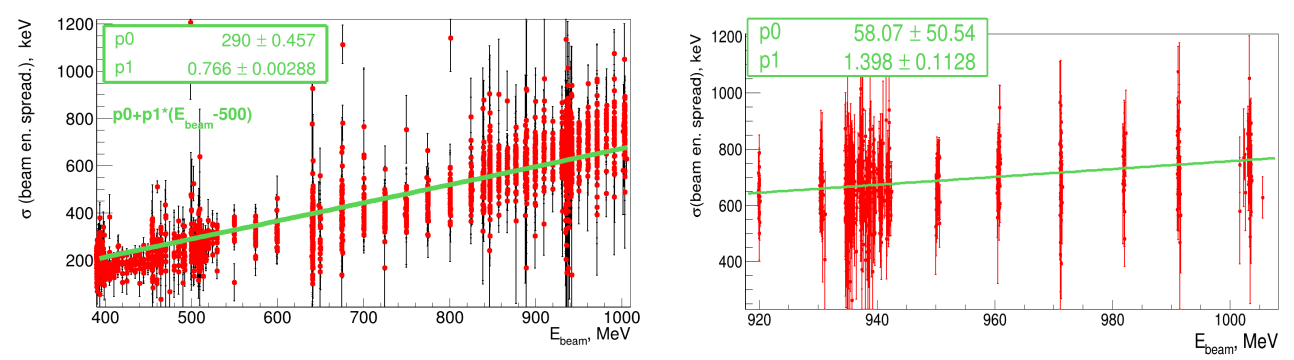

Figure 1. The measured energy spread vs beam energy for a wide energy range (left) and for the beam energies around the $N \bar{N}$ threshold. A linear fit is used for the calculations.

The luminosity was measured using events of Bhabha scattering at large angles [25].

\section{The $e^{+} e^{-} \rightarrow 3\left(\pi^{+} \pi^{-}\right)$cross section}

The analysis of the $e^{+} e^{-} \rightarrow 3\left(\pi^{+} \pi^{-}\right)$process was described in detail in Ref. [8]. For the new data we have reproduced all steps for selection of five and six charged tracks as well as the calculation of the efficiency and radiative corrections. As in Ref. [8], we have a 
background-free sample of the six-track signal events, and use the ratio of the five- and sixtrack events to correct the efficiency. With the new data sample, the number of signal events with six charged tracks increased to 10155 (compared to 2887 events in the previous analysis) and that with one missing track to 17822 (5069) events. The cross section obtained from the new data is shown in Fig. 3 by squares, while the BaBar [6] and previous CMD-3 [8] data are shown by open and closed circles, respectively. Our previous result is confirmed with better statistical accuracy, while a systematic uncertainty is estimated at the same $6 \%$ level, mostly dominated by the uncertainties in the efficiency and background estimate. The "dip" at the $N \bar{N}$ threshold is also confirmed and is studied in more detail (see below).

\section{The $e^{+} e^{-} \rightarrow K^{+} K^{-} \pi^{+} \pi^{-}$cross section}

The analysis of the $e^{+} e^{-} \rightarrow K^{+} K^{-} \pi^{+} \pi^{-}$process was described in detail in Ref. [26]. For the new data we have reproduced all steps for selection of four charged tracks, pion-kaon separation procedure, and the calculation of the efficiency and radiative corrections.

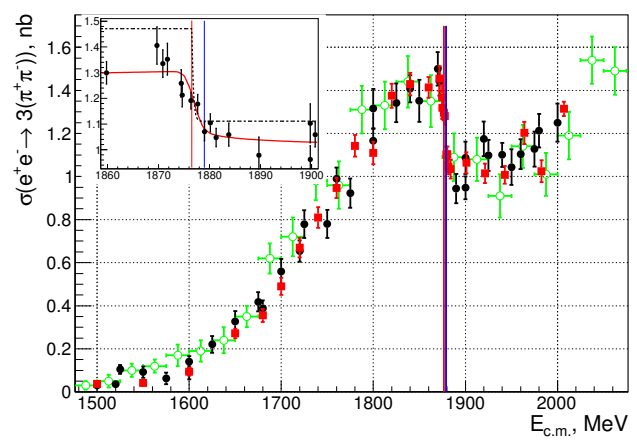

Figure 2. The $e^{+} e^{-} \rightarrow 3\left(\pi^{+} \pi^{-}\right)$Born cross section measured with the CMD-3 detector in the 2017 run (squares). The results of the previous CMD-3 measurement [8] are shown by dots and those of BaBar [6] by open circles. The inset shows the visible cross section with the fit described in the text. The vertical lines show the $N \bar{N}$ thresholds.

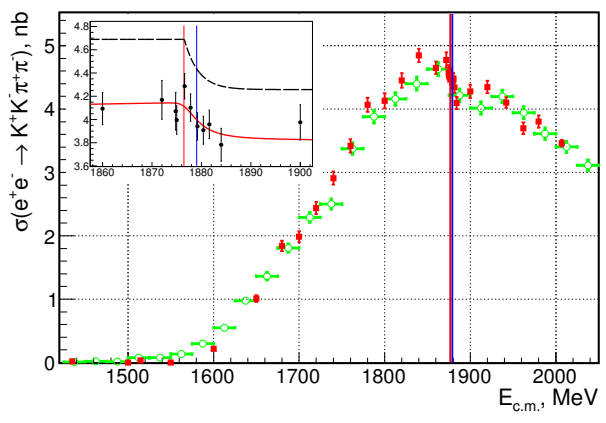

Figure 3. The $e^{+} e^{-} \rightarrow K^{+} K^{-} \pi^{+} \pi^{-}$Born cross section measured with the CMD-3 detector in the 2017 run (squares). The results of the BaBar [27] measurements are shown by open circles. The inset shows the visible cross section with the fit described in the text. The vertical lines show the $N \bar{N}$ thresholds.

A specially designed likelihood function is used to separate kaons and pions. In this analysis we use events with exactly four charged tracks which have practically no background. In contrast to the previous analysis, the events with one missing kaon or events with a missing pion are not used to reduce the uncertainty in the background subtraction. Nevertheless, the same overall statistical accuracy is achieved since the scan around the $N \bar{N}$ threshold is performed with large integrated luminosity that allows us to select about 1500 signal events per energy point. The cross section obtained from the new data is shown in Fig. 3 by squares, while the BaBar [27] data are shown by open circles. Our previous result is confirmed with better statistical accuracy, while a systematic uncertainty remains at the same $6 \%$ level, dominated by uncertainty in the efficiency estimate. Evidence for the "dip" at the $N \bar{N}$ threshold is obtained for the first time in this channel and is studied in more detail below. 


\section{The $e^{+} e^{-} \rightarrow p \bar{p}$ cross section at the $N \bar{N}$ threshold}

The analysis procedure is described in our previous publication [15]. At the energies near threshold, for $\mathrm{E}_{\mathrm{c} . \mathrm{m} .}<1900 \mathrm{MeV}$, protons and antiprotons from the reaction $e^{+} e^{-} \rightarrow$ $p \bar{p}$ stop in the material of the beam pipe because of very low momentum. To select such events, we look for the products of antiproton annihilation with more than two charged tracks coming from the aluminum beam pipe. Comparison of the calorimeter response for such events below and above the $N \bar{N}$ threshold yields the number of $p \bar{p}$ events. Points below the production thresholds, where we assume no signal from the $e^{+} e^{-} \rightarrow p \bar{p}$ reaction, are used for background normalization and we obtain $490 \pm 30$ signal events in the energy range from the production threshold to $1900 \mathrm{MeV}$. Starting from $\mathrm{E}_{\mathrm{c} . \mathrm{m} .}=1900 \mathrm{MeV}$, protons have enough energy to penetrate the beam pipe, and above this energy no annihilation of antiprotons at the beam pipe is observed. Protons and antiprotons are detected as collinear tracks with large specific energy losses, $\mathrm{dE} / \mathrm{dx}$, in the drift chamber (DC) of the CMD-3: we detect 4770 signal events. At each energy a visible cross section is calculated as the number of selected events divided by the detection efficiency and integrated luminosity. The obtained $e^{+} e^{-} \rightarrow p \bar{p}$ visible cross section is shown in Fig. 3. We estimate the systematic uncertainty as about $10 \%$, dominated by the uncertainty in the efficiency calculation: a special study was performed to estimate data-MC difference in the reconstruction efficiency.

\section{The $N \bar{N}$ threshold region}

The cross section in Fig. 3 exhibits very sharp step-like behavior close to the $N \bar{N}$ threshold. The Born cross section cannot be obtained without taking into account its smearing due to radiation of real photons by initial electrons and positrons, and the energy spread of the collision energy with $\sigma_{\mathrm{E}_{\mathrm{c} . \mathrm{m} .}}=0.95 \pm 0.10 \mathrm{MeV}$. The visible cross section is described by a

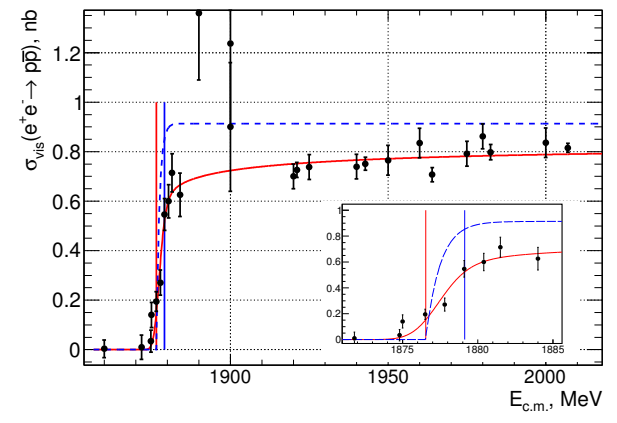

Figure 4. The $e^{+} e^{-} \rightarrow p \bar{p}$ visible cross section measured with the CMD-3 detector. The solid curve shows the result of the fit to an exponentially saturated function of Eq.(3) (shown by the dashed curve) convolved with the $0.95 \mathrm{MeV}$ energy spread and radiation functions. The vertical lines show the $p \bar{p}$ and $n \bar{n}$ thresholds. The inset shows the expanded view of the visible cross section.

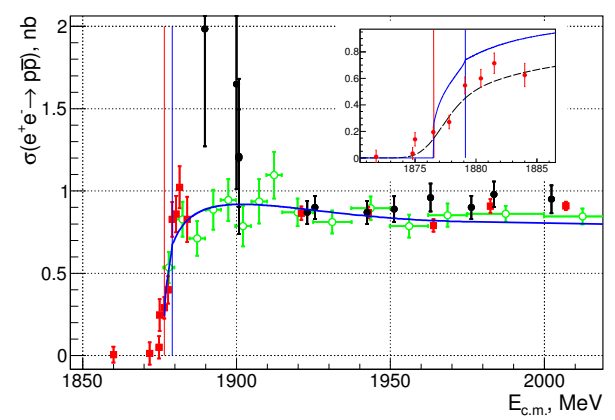

Figure 5. The $e^{+} e^{-} \rightarrow p \bar{p}$ Born cross section measured with CMD-3 (dots [15] and squares) and BaBar (open circles). The solid curve shows the result of the prediction from Refs. [11-13]. The inset shows the expanded view of the visible cross section from CMD-3 with the theoretical prediction for the Born (solid line), and for the visible cross section (dashed line) with the experimental effects. The vertical lines show the $p \bar{p}$ and $n \bar{n}$ thresholds. 
convolution of the radiative cross section, $\sigma_{f \gamma}\left(\mathrm{E}_{\mathrm{c} . \mathrm{m}}\right)$, with the c.m. energy spread function:

$$
\sigma_{\text {vis }}\left(\mathrm{E}_{\mathrm{c} . \mathrm{m} .}\right)=\frac{1}{\sqrt{2 \pi} \sigma_{\mathrm{E}_{\mathrm{c} . \mathrm{m} .}}} \int \mathrm{dE}_{\mathrm{c} . \mathrm{m} .}^{\prime} \sigma_{\mathrm{f} \gamma}\left(\mathrm{E}_{\mathrm{c} . \mathrm{m} .}^{\prime}\right) \cdot \exp \left(-\frac{\left(\mathrm{E}_{\mathrm{c} . \mathrm{m} .}-\mathrm{E}_{\mathrm{c} . \mathrm{m} .}^{\prime}\right)^{2}}{2 \sigma_{\mathrm{E}_{\mathrm{c} . \mathrm{m} .}}^{2}}\right),
$$

where $\sigma_{\mathrm{f} \gamma}\left(\mathrm{E}_{\mathrm{c} . \mathrm{m} .}\right)$ is a convolution of the Born cross section with the radiator function $\mathrm{F}\left(\mathrm{E}_{\mathrm{c} . \mathrm{m} .}, \mathrm{E}_{\gamma}\right)[28,29]$ :

$$
\sigma_{\mathrm{f} \gamma}\left(\mathrm{E}_{\mathrm{c} . \mathrm{m} .}\right)=\int_{0}^{\mathrm{E}_{\gamma}^{\max }} \mathrm{dE}_{\gamma} \cdot \sigma_{\text {Born }}\left(\mathrm{E}_{\mathrm{c} . \mathrm{m} .} \sqrt{1-E_{\gamma} / \mathrm{E}_{\mathrm{c} . \mathrm{m} .}}\right) \cdot \mathrm{F}\left(\mathrm{E}_{\mathrm{c} . \mathrm{m} .}, \mathrm{E}_{\gamma}\right),
$$

where $\mathrm{E}_{\gamma}$ is the radiative photon energy, and $\mathrm{E}_{\gamma}^{\max }$ is a maximum allowed photon energy for the reaction.

For a demonstration of very fast variation of the cross section, $\sigma_{\text {Born }}\left(\mathrm{E}_{\mathrm{c} . \mathrm{m} .}\right)$ is described with an exponentially saturated function,

$$
\sigma_{\text {Born }}\left(\mathrm{E}_{\mathrm{c} . \mathrm{m} .}\right)=\mathrm{A}+\mathrm{B}\left[1-\exp \left(-\frac{\left(\mathrm{E}_{\mathrm{c} . \mathrm{m} .}-\mathrm{E}_{\mathrm{thr}}\right)}{\sigma_{\mathrm{thr}}}\right)\right],
$$

where $\mathrm{E}_{\text {thr }}\left(\mathrm{E}_{\mathrm{c} . \mathrm{m} .}>\mathrm{E}_{\mathrm{thr}}\right)$ and $\sigma_{\mathrm{thr}}$ are the energy threshold and a variation scale of the Born cross section, respectively. The values of $\mathrm{A}$ and $\mathrm{A}+\mathrm{B}\left(\mathrm{B}=0\right.$ for $\left.\mathrm{E}_{\mathrm{c} . \mathrm{m} .}<\mathrm{E}_{\text {thr }}\right)$ give the asymptotic values of the cross section values below and above the $p \bar{p}$ threshold.

We perform a simultaneous fit of all three channels with common $\mathrm{E}_{\mathrm{thr}}$ and $\sigma_{\mathrm{thr}}$ values, and the fit yields $1876.87 \pm 0.10 \mp 0.11 \mathrm{MeV}$ and $0.31 \pm 0.25 \mp 0.15 \mathrm{MeV}$, respectively, with $\chi^{2} / n d f=66 /(67-7)$. The second uncertainty is systematic and anticorrelated with the systematic uncertainty on the energy spread $0.95 \pm 0.10 \mathrm{MeV}$.

Unfortunately, the accelerator-induced energy spread and relatively low statistical accuracy do not allow us to directly observe a possible structure of this rise (drop) due to the proton-neutron interaction, which could be expected in the studied reactions.

In a recently published paper [13], the authors use the optical potential to make a prediction of the $p \bar{p}$ and $n \bar{n}$ cross section behavior at very small energies above the production thresholds. Figure 3 shows good agreement of available data for the $e^{+} e^{-} \rightarrow p \bar{p}$ Born cross section with the theoretical prediction. But for very small deviations from the threshold, energy spread and radiative effects must be taken into account: the result of this convolution for the theoretical function is shown in the inset in comparison with our visible cross section. Note, the suggested model of the final-state interaction of a very slow $N \bar{N}$ pair predicts a nonzero cross section at the $p \bar{p}$ threshold due to the Coulomb interaction, but experimental effects and limited accuracy do not allow us to prove that.

\section{The $e^{+} e^{-} \rightarrow 2\left(\pi^{+} \pi^{-}\right)$cross section at the $N \bar{N}$ threshold}

As suggested in Ref. [13], the total hadronic cross section is strongly affected by virtual production and annihilation of the $N \bar{N}$ pairs. The calculation predicts a $7 \mathrm{nb}$ "bump" in the total cross section, which is about $40 \mathrm{nb}$ at this energy, and should be seen in all $e^{+} e^{-} \rightarrow$ hadrons final states. A naive expectation suggests that the effect could be proportional to the probability of $p \bar{p}$ annihilation into the studied final state.

To test that, we analyze data at the $N \bar{N}$ threshold by selecting events for the reaction $e^{+} e^{-} \rightarrow 2\left(\pi^{+} \pi^{-}\right)$according to the procedure described in Ref. [30], and show the obtained cross section in Fig. 6 together with the most precise measurement by BaBar [31]. While the overall systematic uncertainties on the cross section are still under investigation, the relative 


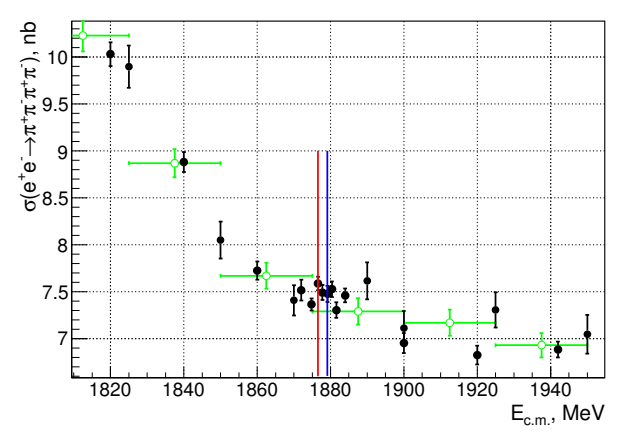

Figure 6. The $e^{+} e^{-} \rightarrow 2\left(\pi^{+} \pi^{-}\right)$cross section measured with CMD-3 (dots) and BaBar [31] (open circles). The vertical lines show the $p \bar{p}$ and $n \bar{n}$ thresholds.

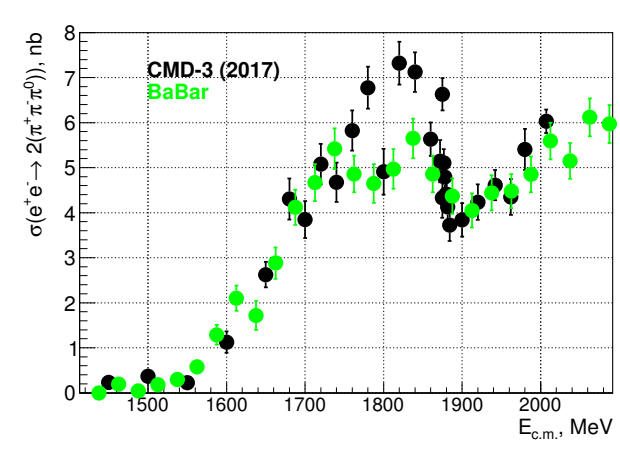

Figure 7. The $e^{+} e^{-} \rightarrow 2\left(\pi^{+} \pi^{-}\right) \pi^{0} \pi^{0}$ cross section measured with CMD-3 (dots, very preliminary) and BaBar [6] (open circles).

point-to-point errors do not exceed 0.1-0.2 nb. No structure exceeding the level of $0.1 \mathrm{nb}$ is observed at the $N \bar{N}$ threshold in either measurement. According to Ref. [32], the probability of $p \bar{p}$ annihilation (with isospin one) to four charged pions is about $14 \%$, while for six charged pions it is about $6 \%$. If a cross section drop in the hadronic channel is related to virtual $N \bar{N}$ annihilation [13], for four-pion production one could expect an about $0.5-0.8 \mathrm{nb}$ drop in the cross section, which is not supported by our data. Note that according to Ref. [32] the probability of $N \bar{N}$ annihilation to the $K^{+} K^{-} \pi^{+} \pi^{-}$final state is much lower than that for six- or four-pion states, and observation of the "dip" in this channel indicates a complicated production dynamics. In contrast, our very preliminary result on the $e^{+} e^{-} \rightarrow 2\left(\pi^{+} \pi^{-}\right) \pi^{0} \pi^{0}$ cross section study confirms the "dip", shown in Fig. 6, while preliminary result for the $e^{+} e^{-} \rightarrow \pi^{+} \pi^{-} 2\left(\pi^{0} \pi^{0}\right)$ cross section, presented by SND group in this Conference [33], shows no effect.

\section{Conclusion}

Using the improved performance of VEPP-2000, the scan of the $e^{+} e^{-}$c.m. energy in the $1680-2007 \mathrm{MeV}$ range has been performed. A detailed study of the $N \bar{N}$ threshold region confirms a fast drop (rise) in the $e^{+} e^{-} \rightarrow 3\left(\pi^{+} \pi^{-}\right)\left(e^{+} e^{-} \rightarrow p \bar{p}\right)$ cross section observed previously. For the first time a width of this structure is measured in the $e^{+} e^{-} \rightarrow p \bar{p}$ reaction: the $\sigma_{\text {thr }}=0.76 \pm 0.28 \mathrm{MeV}$ value is smaller than the difference between the $p \bar{p}$ and $n \bar{n}$ production thresholds. The energy position of the "dip" in the $e^{+} e^{-} \rightarrow K^{+} K^{-} \pi^{+} \pi^{-}$cross sections, observed for the first time, is consistent with the $n \bar{n}$ production threshold, while that for the $e^{+} e^{-} \rightarrow 3\left(\pi^{+} \pi^{-}\right)$reaction is close to the $p \bar{p}$ threshold. No structures in the $e^{+} e^{-} \rightarrow 2\left(\pi^{+} \pi^{-}\right)$ cross section have been found at the $N \bar{N}$ threshold.

\section{References}

[1] R. Baldini et al., reported at the "Fenice" Workshop, Frascati (1988).

[2] A. B. Clegg and A. Donnachie, Z. Phys. C45, 677 (1990).

[3] M.R. Whalley, J. Phys. G29, A1 (2003).

[4] P. L. Frabetti et al. (FOCUS Collaboration), Phys. Lett. B514, 240 (2001).

[5] P. L. Frabetti et al. (FOCUS Collaboration), Phys. Lett. B578, 290 (2004). 
[6] B. Aubert et al. (BaBar Collaboration), Phys. Rev. D73, 052003 (2006).

[7] A. Antonelli et al. (FENICE Collaboration), Phys. Lett. B365, 427 (1996).

[8] R. R. Akhmetshin et al. (CMD-3 Collaboration), Phys. Lett. B723, 82 (2013).

[9] A. Sibirtsev and J. Haidenbauer, Phys. Rev. D71, 054010 (2005).

[10] J. Haidenbauer et al., Phys. Rev. D92, 054032 (2015).

[11] V. F. Dmitriev, A. I. Milstein and S. G. Salnikov, Phys. Rev. D93, 034033 (2016).

[12] A. I. Milstein, S. G. Salnikov, Nucl. Phys. A966, 54 (2017).

[13] A. I. Milstein and S. G. Salnikov, Nucl. Phys. A977, 60 (2018).

[14] B. Aubert et al. (BaBar Collaboration), Phys. Rev. D73, 012005 (2006).

[15] R. R. Akhmetshin et al. (CMD-3 Collaboration), Phys. Lett. B759, 634 (2016).

[16] M. N. Achasov et al. (SND Collaboration), Phys.Rev. D90, 112007 (2014).

[17] M. Ablikim et al. (BESIII Collaboration), Phys. Rev. Lett. 117, 042002 (2016).

[18] B. I. Khazin, Nucl. Phys. B (Proc. Suppl.) 181-182, 376 (2008).

[19] V. V. Danilov et al., Proceedings EPAC96, Barcelona, p.1593 (1996).

[20] I. A. Koop, Nucl. Phys. B (Proc. Suppl.) 181-182, 371 (2008).

[21] P. Yu. Shatunov et al., Phys.Part.Nucl.Lett. 13, 995 (2016).

[22] D. Shwartz et al., PoS ICHEP2016, 054 (2016).

[23] E. V. Abakumova et al., Phys. Rev. Lett. 110, 140402 (2013).

[24] E. V. Abakumova et al., JINST 10, T09001 (2015).

[25] A.E. Ryzhenenkov et al., JINST 12, C07040 (2017).

[26] D. N. Shemyakin et al., (CMD-3 Collaboration), Phys. Lett. B756, 153 (2016).

[27] B. Aubert et al. (BaBar Collaboration), Phys. Rev. D76, 012008 (2007).

[28] E. A. Kuraev and V. S. Fadin, Sov. J. Nucl. Phys. 41, 466 (1985).

[29] S. Actis et al., Eur. Phys. J. C66, 585 (2010).

[30] R. R. Akhmetshin et al. (CMD-3 Collaboration), Phys. Lett. B768, 345 (2017).

[31] B. Aubert et al., (BaBar Collaboration), Phys. Rev. D85, 112009 (2012).

[32] E. Klempt, C. Batty, J.-M. Richard, Phys. Rept. 413, 197 (2005).

[33] M. N. Achasov et al. (SND Collaboration), This Conference. 